\title{
Neutrophilia in locally advanced cervical cancer: A novel biomarker for image-guided adaptive brachytherapy?
}

\author{
Alexandre Escande ${ }^{1}$, Christine Haie-Meder ${ }^{1}$, Pierre Maroun $^{1,2}$, Sébastien Gouy ${ }^{3}$, \\ Renaud Mazeron ${ }^{1}$, Thomas Leroy ${ }^{4}$, Enrica Bentivegna ${ }^{3}$, Philippe Morice ${ }^{2,3,5}$, Eric \\ Deutsch ${ }^{1,2,5}$, Cyrus Chargari ${ }^{1,2,5,6,7}$ \\ ${ }^{1}$ Radiotherapy department, Brachytherapy Unit, Gustave Roussy Cancer Campus, Villejuif, France \\ ${ }^{2}$ Faculté de Médecine PARIS Sud, Université Paris Sud, Université Paris Saclay, France \\ ${ }^{3}$ Department of Surgery, Gustave Roussy, Villejuif, France \\ ${ }^{4}$ Radiotherapy Department, Oscar Lambret Comprehensive Cancer Center, Lille, France \\ ${ }^{5}$ INSERM1030, Gustave Roussy Cancer Campus, Villejuif, France \\ ${ }^{6}$ French Military Health Services Academy, Ecole du Val-de-Grâce, Paris, France \\ ${ }^{7}$ Institut de Recherche Biomédicale des Armées, Bretigny-sur-Orge, France \\ Correspondence to: Cyrus Chargari, email: cyrus.chargari@gustaveroussy.fr
}

Keywords: locally advanced cervical cancer, image-guided adaptive brachytherapy, prognostic factor, biomarkers, neutrophilia

Received: June 27, 2016

Accepted: September 19, 2016

Published: October 04, 2016

\section{ABSTRACT}

Objective: To study the prognostic value of leucocyte disorders in a prospective cohort of cervical cancer patients receiving definitive chemoradiation plus imageguided adaptive brachytherapy (IGABT).

Results: 113 patients were identified. All patients received a pelvic irradiation concomitant with chemotherapy, extended to the para-aortic area in 13 patients with IVB disease. Neutrophilia and leukocytosis were significant univariate prognostic factors for poorer local failure-free survival $(p=0.000$ and $p=0.002$, respectively), associated with tumor size, high-risk clinical target volume (HR-CTV) and anemia. No effect was shown for distant metastases but leukocytosis and neutrophila were both poor prognostic factors for in-field relapses $(p=0.003$ and $p<0.001)$. In multivariate analysis, HR-CTV volume $(p=0.026)$ and neutrophils count $>7,500 / \mu l(p=0.018)$ were independent factors for poorer survival without local failure, with hazard ratio (HR) of 3.1.

Materials and methods: We examined patients treated in our Institution between April 2009 and July 2015 by concurrent chemoradiation (45 Gy in 25 fractions +/lymph node boosts) followed by a magnetic resonance imaging (MRI)-guided adaptive pulse-dose rate brachytherapy ( $15 \mathrm{~Gy}$ to the intermediate-risk clinical target volume). The prognostic value of pretreatment leucocyte disorders was examined. Leukocytosis and neutrophilia were defined as a leukocyte count or a neutrophils count exceeding 10,000 and $7,500 / \mu l$, respectively.

Conclusions: Neutrophilia is a significant prognostic factor for local relapse in locally advanced cervical cancer treated with MRI-based IGABT. This biomarker could help identifying patients with higher risk of local relapse and requiring dose escalation. 


\section{INTRODUCTION}

Despite the implementation of the concept of image-guided adaptive brachytherapy (IGABT) and dose escalation, distant failures remain frequent events in locally advanced cervical cancer, occurring in $30-40 \%$ of patients and partially alleviating the benefit of improving local control [1-7]. There has been an increasing attempt at better identifying tumor related prognostic factors for locally advanced cervical cancers. Most relevant of these factors include tumor size at diagnosis, FIGO stage, lymph node metastases and volume of high-risk clinical target volume (HR-CTV) $[5,7,8]$. However, it is clear that the ability of these conventional tumor-related risk factors to predict risk of tumor relapse and estimate survival is still insufficient. There is need for more sensitive biomarkers to better determine which patients would get the largest benefit from dose escalation (or at the opposite would be candidates for dose de-escalation), but also to potentially identify patients at very high risk of distant failure requiring intensification of systemic treatments.

Tumor-related leukocytosis (TLR), and more particularly neutrophilia, is a paraneoplastic syndrome reported in various malignant advanced tumors types. It was recently suggested that neutrophilia could be associated with poorer overall survival in primarily treated or recurrent cervical cancer patients $[9,10]$. Expression of cancer cellderived granulocyte-colony stimulating factor (G-CSF) is speculated to be one of the causative mechanisms of neutrophilia, through an aberrant paracrine activity involved in expansion and intra-tumor accumulation of myeloid-derived suppressor cells (MDSCs). MDSCs have been implicated in tumor progression by promoting tumor angiogenesis, metastatic process and immune suppression $[11,12]$. Experimentations in murine models have also showed that MDSCs were also associated with higher resistance to radiotherapy [10]. However, the correlation between leucocyte disorders and the risk of local failure has not been examined in locally advanced cervical cancer patients treated according to modern standards.

In the current study, the prognostic significance of systemic leucocyte modifications on survival and on local control was examined in a single center cohort of patients homogeneously treated according to the modern standard based on chemoradiation plus IGABT in the frame of an academic prospective study.

\section{RESULTS}

\section{Patients and tumors}

A total of 113 patients were identified. Median age was 48.3 years (range: 24.7-75.4 years). Histology was squamous cell carcinoma in 95 patients (84.1\%). Median largest tumor size was $4.7 \mathrm{~cm}$ (range: $1-12 \mathrm{~cm}$ ). Initial median tumor volume assessed by MRI was $39.7 \mathrm{~cm}^{3}$ (range: $\left.10.5-340 \mathrm{~cm}^{3}\right)$. Forty-five patients $(39.8 \%)$ had pelvic nodal uptakes at PET/CT and 13 patients had paraaortic lymph node metastases including five patients with PET positive lymph node metastases and eight patients with histological evidence of para-aortic lymph node metastases. At first week of EBRT, median hemoglobin level was $12.6 \mathrm{~g} / \mathrm{dL}(6.5-15.3 \mathrm{~g} / \mathrm{dL})$ and median platelet count was $286 \mathrm{G} / \mathrm{L}(110-751 \mathrm{G} / \mathrm{L})$. Median leukocytes and neutrophils counts were $8,800 / \mathrm{mm}^{3}\left(3,700-22,500 / \mathrm{mm}^{3}\right)$ and $5,650 /$ $\mathrm{mm}^{3}\left(400-18,700 / \mathrm{mm}^{3}\right)$, respectively. Leukocytosis and neutrophilia were found in 28 patients $(24.8 \%)$ both. Median lymphocyte count was $1,400 / \mathrm{mm}^{3}(200-8,200 /$ $\left.\mathrm{mm}^{3}\right)$. Neutrophilia at initiation of chemoradiation was associated with a larger tumor at diagnosis $(p=0.033)$, and a larger HR-CTV volume at time of brachytherapy $(p=0.012)$. Characteristics of patients and tumors and blood cell count results are shown in Table 1.

\section{Treatment}

All patients received a pelvic irradiation, extended to the para-aortic area in 13 patients with stage IVB disease. All patients received concurrent chemotherapy, consisting of cisplatin in 102 patients $(90.3 \%)$, carboplatin in six, cisplatin followed by carboplatin because of renal function impairment in five (5.4\%). Median dose of sequential boost to macroscopically involved lymph node metastases was 10 Gy (range: 7.2-14 Gy, 1.8-2 Gy per fraction). Median HR-CTV volume was $21.3 \mathrm{~cm}^{3}$ (range: $10-79 \mathrm{~cm}^{3}$ ). The median D90 HR-CTV was $38.2 \mathrm{~Gy}$ (range: 8.4-50.5 Gy). Ten patients $(8.8 \%)$ received a parametrial boost using interstitial needles. Median overall treatment time was 47 days (range: 38-62 days). Treatment characteristics are reported in Table 2.

\section{Outcome}

With median follow-up of 4.1 years (95\% CI: $3.47-$ 4.7 years), relapses were reported in 29 patients $(25.7 \%)$. In details, in-field relapses occurred in 16 patients, including local relapses in 12 patients $(10.6 \%)$. Out-of field relapses occurred in 22 patients $(19.5 \%)$, including six patients with para-aortic failure who had not received para-aortic irradiation at time of primary treatment because primary staging showed no para-aortic metastases. At last followup, 20 patients (17.7\%) had died, all from cancer. At three years, estimated overall survival was $80.0 \%$ (95\% CI: $75.7-$ $84.3 \%)$, estimated relapse-free survival was $73.8 \%$ (95\% CI: $69.3-78.3 \%$ ), estimated survival without in-field relapse $85.6 \%$ (95\% CI: $82-89.2 \%$ ) and estimated survival without local relapse was $89.0 \%$ (95\% CI: 85.8-92.2\%)

\section{Prognostic value of leucocytes disorders}

Using univariate analysis, pre-treatment neutrophilia and leukocytosis were significant prognostic factors for local failure-free survival $(p<0.001$ and $p=0.002$, 
Table 1: Patients, tumors and blood cell count characteristics

\begin{tabular}{|c|c|c|}
\hline Characteristics & & Median (min-max) or $\mathrm{Nb}(\%)$ \\
\hline Number of patients & & $113(100)$ \\
\hline Age (years) & & $48.3(24.7-74.4)$ \\
\hline \multirow[t]{3}{*}{ Performance status } & 0 & $85(75.2)$ \\
\hline & 1 & $26(23.0)$ \\
\hline & 2 & $2(1.8)$ \\
\hline \multirow[t]{2}{*}{ Histopathological type: } & SCC & $95(84.1)$ \\
\hline & ADK & $18(15.9)$ \\
\hline Tumor volume $\left(\mathrm{cm}^{3}\right)$ & & $39.7(10.5-340)$ \\
\hline Largest tumor size $(\mathrm{cm})$ & & $4.7(1-12)$ \\
\hline \multirow[t]{2}{*}{ Maximal size $>5 \mathrm{~cm}$} & Yes & $61(46.0)$ \\
\hline & No & $52(54.0)$ \\
\hline \multirow[t]{7}{*}{ FIGO staging } & IB2 & $33(29.2)$ \\
\hline & IIA & $7(6.2)$ \\
\hline & IIB & $51(45.1)$ \\
\hline & IIIA & $1(0.9)$ \\
\hline & IIIB & $3(2.7)$ \\
\hline & IVA & $5(4.4)$ \\
\hline & IVB & $13(11.5)$ \\
\hline \multirow[t]{2}{*}{ Pelvic nodal metastases } & Yes & $45(39.8)$ \\
\hline & No & $68(60.2)$ \\
\hline \multirow[t]{4}{*}{ Differentiation degree } & Well & $27(23.9)$ \\
\hline & Moderate & $42(37.2)$ \\
\hline & Poor & $17(15)$ \\
\hline & ND & $27(23.9)$ \\
\hline \multicolumn{3}{|l|}{ Blood cell count at first week } \\
\hline Hemoglobin $(\mathrm{g} / \mathrm{dl})$ & & $12.6(6.5-15.3)$ \\
\hline Platelet $(\mathrm{G} / \mathrm{L})$ & & $286(110-751)$ \\
\hline Leucocytes $\left(/ \mathrm{mm}^{3}\right)$ & & $8,800(3,700-22,500)$ \\
\hline Neutrophils $\left(/ \mathrm{mm}^{3}\right)$ & & $5,650(400-18,700)$ \\
\hline \multirow[t]{2}{*}{ Leucocytosis } & Yes & $28(24.8)$ \\
\hline & No & $85(75.2)$ \\
\hline \multirow[t]{2}{*}{ Neutrophilia } & Yes & $28(24.8)$ \\
\hline & No & $85(75.2)$ \\
\hline
\end{tabular}

ADK: adenocarcinoma; FIGO: International Federation of Gynecology Obstetrics; SCC: squamous cell carcinoma; $\mathrm{Nb}$ : number.

respectively), associated with tumor size $(p=0.003)$, HR-CTV volume $(p=0.003)$ and anemia $(p=0.036)$. The effect of leukocytosis or neutrophila was not significant for distant failures but leukocytosis and neutrophila were both poor prognostic factors for in-field relapse $(p=0.003$ and $p<0.001)$, associated with HR-CTV volume $(p=0.008)$. In univariate analysis significant factors for overall survival were tumor size $(p=0.01)$, HR-CTV volume $(p=0.017)$, neutrophilia $(p=0.004)$ and anemia $(p=0.011)$.
Using multivariate analysis, HR-CTV volume $(p=0.026)$ and neutrophils count $>7,500 / \mu \mathrm{l}(p=0.018)$ were independent factors for poorer survival without local failure, with hazard ratio (HR) of 3.1 for both factors. Two prognostic factors were associated with a higher risk of in-field failure: $1 /$ leukocyte disorders (for neutrophilia: $\mathrm{HR}=4.50, p=0.002$; for leukocytosis: $\mathrm{HR}=2.54, p=0.047)$ and $2 / \mathrm{a}$ HR-CTV volume $>25 \mathrm{cc}$ $(\mathrm{HR}=2.94, p=0.034)$. In multivariate, only HR-CTV 
Table 2: Treatments characteristics

\begin{tabular}{|l|c|}
\hline \multicolumn{1}{|c|}{ Characteristics } & Median (min-max) or Nb(\%) \\
\hline Number of patients & $113(100)$ \\
\hline Radiotherapy & 45 \\
\hline Dose (Gy) & $113(100)$ \\
\hline Pelvic irradiation & $13(11.5)$ \\
\hline Para-aortic irradiation & $45(39.8)$ \\
\hline Sequential lymph node boosts & $10(7.2-14)$ \\
\hline Median boosts doses (Gy) & $113(100)$ \\
\hline Yes & $0(0)$ \\
\hline Concurrent chemotherapy Carboplatine & $6(3-7)$ \\
\hline Number of chemotherapy courses & $102(90.3)$ \\
\hline Chemotherapy type & $6(5.3)$ \\
\hline \multicolumn{1}{|c|}{ Cisplatine } & $5(4.4)$ \\
\hline Brachytherapy parameters & $113(100)$ \\
\hline MRI guided & $21.3(9.9-79.0)$ \\
\hline HR-CTV volume (cm $\left.{ }^{3}\right)$ & $38.2(17.8-50.5)$ \\
\hline HR-CTV D90 (Gy) & $1.73(0.67-2.43)$ \\
\hline TRAK & $10(8.8)$ \\
\hline Use of interstitial needles & $47(38-62)$ \\
\hline Overall treatment time (days) & \\
\hline
\end{tabular}

HR-CTV: high-risk clinical target volume; MRI: Magnetic Resonance Imaging; TRAK: Total reference air Kerma.

volume $(p=0.03)$ and anemia $(p=0.026)$ were significant for overall survival. The significant prognostic value of neutrophilia and leuckocytosis on local-failure free survival remained significant in multivariate analysis after exclusion of patients with stage IVB disease (HR $=8.9$ and 6.7, respectively, $p=0.002$ for both). Results of univariate and multivariate analyses are detailed in Table 3. Local failure-free survival curve according to presence or absence of neutrophilia is shown in Figure 1.

\section{DISCUSSION}

The progressive implementation of IGABT in locally advanced cervical cancer patients has been associated with a significant increase of local control rates in patients with large tumors at diagnosis [1-7]. Pötter et al. reported their retrospective experience comparing 2D brachytherapy and MRI-guided brachytherapy. The impact of IGABT in tumors less than $5 \mathrm{~cm}$ could not be evidenced but for tumors $>5 \mathrm{~cm}$, the use IGABT was associated with an increase in the mean D90 HR-CTV and an improvement of 3-year local control rate from $64 \%$ to $82 \%$. The decrease of local relapses was associated with a survival benefit in patients with bulky tumors, from $53 \%$ to $64 \%$ [3]. In our previously published experience of IGABT, we had reported that the only tumor-related factor associated with local control in multivariate analysis was HR-CTV volume, which reflects a bulky tumor at diagnosis but also poor response to chemoradiation. Analysis of dose-effect curves suggested that doses to the HR-CTV should be increased in higher stage or in patients with a HR-CTV volume $>30 \mathrm{~cm}^{3}$ [3]. Although there is sound rationale that dose escalation improves local control in patients with bulky disease, there is no firm demonstration that dosimetric parameters and survival are correlated. It is therefore uncertain whether all patients equally benefit from dose escalation and the possibility that some patients would not benefit from dose escalation cannot be ruled out. Some tumoral factors (FIGO stage, width at diagnosis, treatment time, HR-CTV volume, anemia) have been shown to significantly impact dose volume effect for tumor control, but in the next steps of treatment optimization, other parameters should be taken into account in the treatment planning process, such as tumor biological heterogeneity or tumor response after chemoradiation $[5,7,8]$.

Biological parameters could be added to current models for prediction of outcome and tailoring therapeutic strategies based on a personalized assessment of specific risk. Mayr et al. have monitored treatment response by functional MRI and their results suggest that tumor heterogeneity could be characterized by dynamic contrast- 
Table 3: Results of univariate and multivariate analyses

\begin{tabular}{|c|c|c|c|c|}
\hline \multirow[t]{2}{*}{ variable } & \multicolumn{2}{|c|}{ Overall Survival } & \multicolumn{2}{|c|}{ In-Field Failure-Free Survival } \\
\hline & Log-rank & Cox model (HR) & Log-rank & Cox model (HR) \\
\hline FIGO III-IV & 0.959 & -- & 0.552 & -- \\
\hline Tumor $>5 \mathrm{~cm}$ & 0.010 & 0.512 & 0.002 & 0.078 \\
\hline Pelvic LN & 0.151 & -- & 0.099 & 0.288 \\
\hline $\mathrm{HR}-\mathrm{CTV}>25 \mathrm{cc}$ & 0.017 & $0.030(2.86)$ & 0.002 & $0.008(3.73)$ \\
\hline Neutrophilia $^{\mathrm{a}}$ & 0.004 & 0.232 & 0.000 & $0.002(4.50)$ \\
\hline Leucocytosis $^{\mathrm{a}}$ & 0.199 & 0.962 & 0.003 & $0.047(2.54)$ \\
\hline Anemia & 0.011 & $0.026(3.05)$ & 0.065 & 0.233 \\
\hline \multirow[t]{2}{*}{ Variable } & \multicolumn{2}{|c|}{ Local Failure-Free Survival } & \multicolumn{2}{|c|}{ Distant Failure-Free Survival } \\
\hline & Log-rank & Cox model (HR) & Log-rank & Cox model (HR) \\
\hline FIGO III-IV & 0.669 & -- & 0.433 & -- \\
\hline Tumor $>5 \mathrm{~cm}$ & 0.003 & 0.383 & 0.013 & $0.005(3.48)$ \\
\hline Pelvic LN & 0.914 & -- & 0.047 & $0.028(2.42)$ \\
\hline HR-CTV $>25 \mathrm{cc}$ & 0.003 & $0.026(3.1)$ & 0.075 & 0.505 \\
\hline Neutrophilia $^{a}$ & 0.000 & $0.018(3.1)$ & 0.590 & -- \\
\hline Leucocytosis $^{\mathrm{a}}$ & 0.002 & 0.287 & 0.564 & -- \\
\hline Anemia & 0.036 & 0.177 & 0.020 & 0.324 \\
\hline
\end{tabular}

Variables significant with a $p$ value $<0.1$ in univariate analysis were used for multivariate model (significant factors in multivariate analysis in bold) .

FIGO: International Federation of Gynecology Obstetrics; HR: hazard ratio (given only if $p<0.05$ ); HR CTV: high risk clinical target volume; LN: lymph nodes.

a, Neutrophilia and leucocytosis were not tested in the same model, as neutrophils are subpopulation of leucocytes.

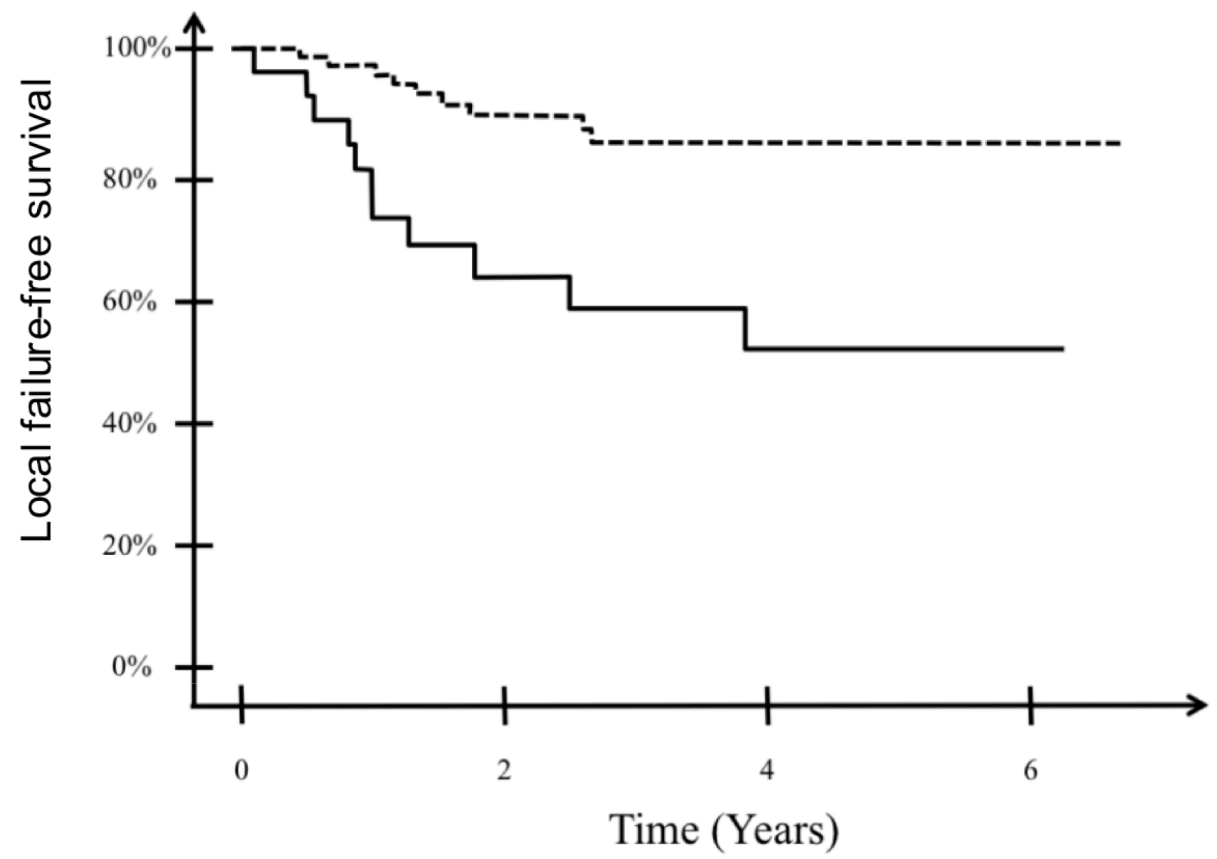

Dotted line for patients with $P N N<7,500 / \mu 1$

Full line for patients with $P N N \geq 7,500 / \mu 1$

Figure 1: Estimated survival without local failure in patients with or without neutrophilia. 
enhanced to predict long-term treatment outcome, including the probability of primary tumor control [13]. Anemia is another factor that is frequently reported in the literature, with retrospective analyses suggesting that hemoglobin level during radiotherapy is a strong prognostic factor for local control and survival and a biomarker that could potentially allow identifying poor prognostic subgroups with persisting hypoxia during radiotherapy [14]. The prognostic value of anemia was corroborated in the present study.

Leukocyte disorders have recently gained some interest as biomarkers, following data reporting leukocytosis would occur in $1-10 \%$ of patients with nonhematopoietic malignancies and would be associated with a poorer prognosis [9, 15-17]. Leukocytosis is caused by upregulation of expression of hematological growth factors produced by tumors. Those, which act as autocrine stimulating agents, include granulocyte colony-stimulating growth factors, granulocyte macrophage colony-stimulating growth factor, interleukins (1 and 6) and tumor-necrosis factor alpha $(\mathrm{TNF} \alpha)[16,18,19]$. Mabuchi et al. examined the prognostic value of leukocytosis in a cohort of cervical cancer patients. They retrospectively found that a white blood cells count $\geq 10,000 / \mu$ l was associated with more frequent treatment failures and shorter survival. They also reported that tumors from patients with leukocytosis had stronger immunoreactivity for G-CSF than those obtained from patients without leukocytosis. Leukocytosis was a significant prognostic factor for OS in multivariate analysis. This effect on survival was confirmed in a prospective study showing that patients with leukocytosis had more frequent treatment failures, shorter PFS and higher serum G-CSF concentration than those without leukocytosis. When examining leukocyte subtypes, all patients with leukocytosis had elevated neutrophils count, but no increase in basophils or monocytes, indicating that leukocytosis is a result of elevated neutrophils count [9]. More recently, further preclinical investigations were performed to examine the mechanisms responsible for tumor-related leukocytosis in cervical cancer, showing that the increased expression of tumor-derived G-CSF in some cervical cancer was associated with increased frequency of circulating MDSCs in the blood. These cells, which contribute to tumor angiogenesis, metastatic process and immune suppression, could be responsible for the rapidly progressive and radioresistant phenotype of tumor with neutrophilia $[11,12,18]$. Leukocytosis could also reflect a chronic oncogenic inflammation. Actors of this inflammatory process, such as tumor-associated macrophages (TAMs) or MDSCs and their secreted cytokines (IL-6, IL-1 $\beta$, TNF) are now considered as key in promoting tumor progression [11]. In advanced tumors or in hypoxic regions of tumor stroma, TAMs polarize to type 2 macrophages inhibiting anticancer immunity through the release of factors encouraging recruitment of regulatory $\mathrm{T}$ cells as well as production of transforming growth factor $\beta 1$ (TGF- $\beta$ ) [11]. Furthermore, it has been shown a radiation-induced accumulation of TAMs within 1-2 weeks after treatment, partially as a result of increased levels of the transcription factor HIF-1 (hypoxia inducible factor -1$)$, potentially resulting in the increased production of proangiogenic factors involved in vascular network recovery and tumor regrowth after irradiation [20].

Although previously described clinical studies provided meaningful information on the prognostic value of tumor-related neutrophilia and the underlying biological mechanisms, they did not allow drawing conclusions on the possibility to integrate this biological information in the treatment process. Patterns of failure were not examined and authors did not examine whether patients experienced more local relapses or more distant metastatic events. Moreover, patients with metastatic disease were included and treatments were not conducted according to modern standards based on chemoradiation plus IGABT for locally advanced cancers, as stage IB2 - IIB carcinomas were treated with upfront radical hysterectomy $+/$ - adjuvant radiotherapy. Finally, no data was provided on technique of brachytherapy and the effect of HR-CTV volume was not examined, while it is a major prognostic factor of patients' outcome in terms of local relapses as well as in terms of distant failures $[9,10,21]$. Consequently, it was not possible, based on previous data from literature, to have any indication on how leukocytosis might be included as part of the decision process in patients who are treated with IGABT.

In the present study, with all patients being prospectively treated with curative intent according to a modern strategy of dosimetric optimization based on MRI findings, we found that neutrophilia was a major prognostic factor for local failures in multivariate analysis, even after taking into account the three main well known prognostic factors for local failure that are tumor size at diagnosis, HR-CTV volume and anemia. The systematic delivery of concurrent chemotherapy and the implementation of dose escalation could account for the lack of significant effect on survival in multivariate analysis, contrary to previous reports examining the prognostic significance of neutrophilia [9]. To our best knowledge, this is the first study reporting the prognostic value of neutrophilia in terms of local relapse. The effect of the D90 HR-CTV was not significant but we have previously shown a significant correlation between the D90 HR-CTV and the risk of local failure in a larger cohort of 225 patients, with a higher statistical power to show this effect [5]. The lack of correlation between dosimetric parameters and probability of local control could be also explained by an advanced optimization process that had been performed in this homogeneous cohort, with a high median D90 HR-CTV dose in most patients. The correlation between the risk of local relapse and presence of neutropenia suggests that neutrophilia could be a valuable and affordable biomarker for improving selection of appropriate candidates to dose escalation, because of a higher risk for local relapse. 
Another perspective is to conceive pharmacological strategies based on modulation of the tumor immune micro environment to potentiate radiotherapy in these high risk patients, who exhibit radiation-resistant tumors [22, 23]. However, these findings still remain to be confirmed in an independent prospective cohort before applying these concepts to clinics [24]. In particular, the possibility to modulate treatment delivery according to neutrophilia, through dose escalation or dose de-escalation, has not been validated yet.

\section{MATERIALS AND METHODS}

\section{Patients and tumors}

We examined clinical records of consecutive patients treated in our institution between April 2009 and July 2015 for a histologically confirmed locally advanced cervical cancer and receiving concurrent chemoradiation followed by magnetic resonance imaging (MRI)-guided adaptive pulse-dose rate (PDR) brachytherapy within the frame of international prospective observational study examining the effects of MRI-guided brachytherapy in locally advanced cervical cancer: EMBRACE. EMBRACE study aims at assessing MRI-guided brachytherapy for cervical cancer and examining dosimetric parameters of importance for local control and morbidity. All patients had 18-Fluorodeoxyglucose Positron Emission Tomography/ Computed tomography (PET/CT) as part of their primary staging. Those without evidence of para-aortic lymph node metastases underwent a primary laparoscopic para-aortic lymphadenectomy.

\section{Treatment characteristics}

Patients received pelvic external beam radiotherapy (EBRT), 45 Gy in 25 fractions of 1.8 Gy through a 3D conformal technique with high megavoltage photons of a linear accelerator. Patients with PET positive paraaortic lymph nodes or histological evidence of para-aortic metastases received extended field radiotherapy. Patients with visceral metastases were excluded. Concurrent chemotherapy was delivered, cisplatin $40 \mathrm{mg} / \mathrm{m}^{2}$ weekly. Carboplatin AUC (Area Under Curve) 2 was preferred in case of contraindication to cisplatin.

The PDR brachytherapy boost was based on a MRI computerized assisted treatment planning The vaginal mold technique was used, as previously described [25]. Definitions of volumes followed the guidelines of GEC-ESTRO (Groupe Européen de Curiethérapie, European Society for Radiotherapy \& Oncology) $[26,27]$. Brachytherapy was delivered through one single application per patient. When endocavitary implantation was not sufficient for an adequate coverage of HR-CTV, an additional implantation could be performed with interstitial implantation. Treatment planning objective was to deliver at least 60 Gy to $90 \%$ of the intermediate risk clinical target volume (IR-CTV), taking into account the dose delivered by EBRT after converting doses into biological effective doses normalized to a radiobiologically weighted dose equivalent of $2 \mathrm{~Gy} /$ fraction $(\alpha / \beta=10 \mathrm{~Gy})$, and 85 Gy to the D90 of the HR-CTV. Further details on the brachytherapy procedure have been previously reported $[9,12,25,26]$.

Patients with PET positive pelvic or para-aortic lymph nodes received a sequential fractionated boost to deliver 60 Gy to macroscopic lymph nodes, taking into account the contribution of IGABT. The overall treatment time was aimed at not exceeding 55 days.

\section{Definition of leukocyte disorders}

Patients underwent systematic complete blood cell counts weekly during chemoradiation. As this is retrospective analysis, assays were performed blinded to the study end point. Pretreatment blood samples taken before any chemotherapy were employed in the current analysis. Leukocytosis and neutrophilia were defined as a leukocyte count or a neutrophils count exceeding 10,000 and $7,500 / \mu 1$, respectively. Anemia was defined with threshold of $12 \mathrm{~g} / \mathrm{dL}$. These cutpoints were defined from usual thresholds used routinely for defining these blood cell counts disorders. Were excluded from analysis patients who received neoadjuvant chemotherapy, corticosteroids, presenting chronic inflammation, patients treated for an immune disease, or presenting acute or chronic infection (including Human Immunodeficiency Virus).

\section{Follow-up and statistical analysis}

Follow-up was scheduled at 6 weeks, then every three months during two years. A systematic MRI of the pelvis was performed 6-8 weeks after IGABT, then every six months. Hysterectomy was performed only in case of isolated local failure and after a complete restaging through PET/CT. All relapses were considered (not only first relapse) and classified as local failure occurring in the true pelvis, in-field relapses occurring within pelvic and/or para-aortic EBRT fields (if para-aortic area was irradiated), or out-of field relapse (distant metastatic failure). Factors associated with tumor relapse were examined. Survival times were calculated from the time of histological diagnosis and survival rates were estimated using the Kaplan Meier method. Univariate analyses were carried out using log rank tests. Interaction between factors was tested. Multivariate analyses were performed for variables with $p$ value $<0.2$ in univariate analysis, according to the Cox method. Statistical analyses were performed using SPSS statistics 20.0 ${ }^{\circledR}$ (Statistical Package for Social Science) for windows (an IBM company software, Chicago, Illinois, USA). 


\section{CONCLUSIONS}

In this cohort of patients treated homogeneously according to modern standards, we found that neutrophilia was a significant prognostic factor for local relapse in locally advanced cervical cancer treated with MRI-based IGABT. This biomarker could be used in further clinical trials to better identify patients with higher risk of local relapse and requiring dose escalation and/or concurrent administration of biological modifiers.

\section{CONFLICTS OF INTEREST}

None.

\section{REFERENCES}

1. Pötter R, Georg P, Dimopoulos JC, Grimm M, Berger D, Nesvacil N, Georg D, Schmid MP, Reinthaller A, Sturdza A, Kirisits C. Clinical outcome of protocol based image (MRI) guided adaptive brachytherapy combined with 3D conformal radiotherapy with or without chemotherapy in patients with locally advanced cervical cancer. Radiother Oncol. 2011; 100:116-23.

2. Mazeron R, Gilmore J, Dumas I, Champoudry J, Goulart J, Vanneste B, Tailleur A, Morice P, Haie-Meder C. Adaptive 3D image guided brachytherapy: a strong argument in the debate on systematic radical hysterectomy for locally advanced cervical cancer. Oncologist. 2013; 18:415-22.

3. Pötter R, Dimopoulos J, Georg P, Waldhäusl C, WachterGerstner N, Weitmann H, Reinthaller A, Knocke TH, Wachter S, Kirisits C. Clinical impact of MRI assisted dose volume adaptation and dose escalation in brachytherapy of locally advanced cervix cancer. Radiother Oncol. 2007; 83:148-155.

4. Lindegaard JC, Fokdal LU, Nielsen SK, Juul-Christensen J, Tanderup K. MRI-guided adaptive radiotherapy in locally advanced cervical cancer from a Nordic perspective. Acta Oncol. 2013; 52:1510-9.

5. Mazeron R, Castelnau-Marchand P, Dumas I, del Campo ER, Kom LK, Martinetti F, Farha G, Tailleur A, Morice P, Chargari C, Lefkopoulos D, Haie-Meder C. Impact of treatment time and dose escalation on local control in locally advanced cervical cancer treated by chemoradiation and image-guided pulsed-dose rate adaptive brachytherapy. Radiother Oncol. 2015; 114:257-63.

6. Chargari C, Magné N, Dumas I, Messai T, Vicenzi L, Gillion N, Morice P, Haie-Meder C. Physics and clinical contribution with MRI-based Pulsed dose rate brachytherapy in patients with cervix carcinoma. Int J Radiat Oncol Biol Phys. 2009; 74:133-139.

7. Schmid MP, Franckena M, Kirchheiner K, Sturdza A, Georg P, Dörr W, Pötter R. Distant metastasis in patients with cervical cancer after primary radiotherapy with or without chemotherapy and image guided adaptive brachytherapy. Gynecol Oncol. 2014; 133:256-62.
8. Kim SM, Choi HS, Byun JS. Overall 5-year survival rate and prognostic factors in patients with stage IB and IIA cervical cancer treated by radical hysterectomy and pelvic lymph node dissection. Int J Gynecol Cancer. 2000; 10:305-312.

9. Mabuchi S, Matsumoto Y, Isohashi F, Yoshioka Y, Ohashi H, Morii E, Hamasaki T, Aozasa K, Mutch DG, Kimura T. Pretreatment leukocytosis is an indicator of poor prognosis in patients with cervical cancer. Gynecol Oncol. 2011; 122:25-32.

10. Mabuchi S, Matsumoto Y, Hamasaki T, Kawano M, Hisamatsu T, Mutch DG, Kimura T. Elevated white blood cell count at the time of recurrence diagnosis is an indicator of short survival in patients with recurrent cervical cancer. Int J Gynecol Cancer. 2012; 22:1545-51.

11. Zamarron BF, Chen W. Dual roles of immune cells and their factors in cancer development and progression. Int J Biol Sci. 2011; 7:651-658.

12. Nagaraj S, Gabrilovich DI. Myeloid-derived suppressor cells in human cancer. Cancer J. 2010; 16:348-353.

13. Mayr NA, Huang Z, Wang JZ, Lo SS, Fan JM, Grecula JC, Sammet S, Sammet CL, Jia G, Zhang J, Knopp MV, Yuh WT. Characterizing tumor heterogeneity with functional imaging and quantifying high-risk tumor volume for early prediction of treatment outcome: cervical cancer as a model. Int J Radiat Oncol Biol Phys. 2012; 83:972-9.

14. Dunst J, Kuhnt T, Strauss HG, Krause U, Pelz T, Koelbl H, Haensgen G. Anemia in cervical cancers: impact on survival, patterns of relapse, and association with hypoxia and angiogenesis. Int J Radiat Oncol Biol Phys. 2003; $56: 778-87$.

15. Granger JM, Kontoyiannis DP. Etiology and outcome of extreme leukocytosis in 758 nonhematologic cancer patients: a retrospective, single-institution study. Cancer. 2009; 115:3919-3923.

16. Wilcox RA. Cancer-associated myeloproliferation: old association, new therapeutic target. Mayo Clin Proc 2010; 85:656-663.

17. Matsumoto Y, Mabuchi S, Muraji M, Morii E, Kimura T. Squamous cell carcinoma of the uterine cervix producing granulocyte colony-stimulating factor: a report of 4 cases and a review of the literature. Int J Gynecol Cancer. 2010; 20:417-421.

18. Mabuchi S, Matsumoto $\mathrm{Y}$, Kawano M, Minami K, Seo Y, Sasano T, Takahashi R, Kuroda H, Hisamatsu T, Kakigano A, Hayashi M, Sawada K, Hamasaki T. Uterine cervical cancer displaying tumor-related leukocytosis: a distinct clinical entity with radioresistant feature. J Natl Cancer Inst. 2014; 19:106.

19. Connor JP. Aggressive carcinosarcoma of the uterine cervix associated with high levels of granulocyte colony stimulating factor: case report and laboratory correlates. Gynecol Oncol. 2006; 103:349-53.

20. Jeffery S. Russell, J. Martin Brown. The irradiated tumor microenvironment: role of tumor-associated macrophages in vascular recovery. Front Physiol. 2013; 4:157. 
21. Chargari C, Mazeron R, Escande A, Maroun P, Dumas I, Martinetti F, Tafo-Guemnie A, Deutsch E, Morice P, HaieMeder C. Image-guided adaptive brachytherapy in cervical cancer: Patterns of relapse by brachytherapy planning parameters. Brachytherapy in press.

22. Chargari C, Magne N, Guy JB, Rancoule C, Levy A, Goodman KA, Deutsch E. Optimize and refine therapeutic index in radiation therapy: Overview of a century. Cancer Treat Rev. 2016; 45:58-67.

23. Levy A, Chargari C, Marabelle A, Perfettini JL, Magné N, Deutsch E. Can immunostimulatory agents enhance the abscopal effect of radiotherapy? Eur J Cancer. 2016; 62:36-45.

24. McShane LM, Altman DG, Sauerbrei W, Taube SE, Gion M, Clark GM. Statistics Subcommittee of the NCIEORTC Working Group on Cancer Diagnostics. REporting recommendations for tumour MARKer prognostic studies (REMARK). Br J Cancer. 2005; 93:387-91.

25. Magné N, Chargari C, SanFilippo N, Messai T, Gerbaulet A, Haie-Meder C. Technical aspects and perspectives of the vaginal mold applicator for brachytherapy of gynecological malignancies. Brachytherapy. 2009; 9:274-7.
26. Pötter R, Haie-Meder C, Van Limbergen E, Barillot I, De Brabandere M, Dimopoulos J, Dumas I, Erickson B, Lang S, Nulens A, Petrow P, Rownd J, Kirisits C. Recommendations from gynaecological (GYN) GEC ESTRO working group (II): concepts and terms in $3 \mathrm{D}$ image-based treatment planning in cervix cancer brachytherapy-3D dose volume parameters and aspects of 3D image-based anatomy, radiation physics, radiobiology. Radiother Oncol. 2006; 78:67-77.

27. Haie-Meder C, Pötter R, Van Limbergen E, Briot E, De Brabandere M, Dimopoulos J, Dumas I, Hellebust TP, Kirisits C, Lang S, Muschitz S, Nevinson J, Nulens A. Recommendations from Gynaecological (GYN) GECESTRO Working Group (I): concepts and terms in 3D image based 3D treatment planning in cervix cancer brachytherapy with emphasis on MRI assessment of GTV and CTV. Radiother Oncol. 2005; 74:235-45. 